\title{
Completude do Sistema de Informação em Tuberculose no estado do Paraná, 2008-2017: estudo ecológico
}

\author{
Completeness of the tuberculosis information system in the state of Paraná, 2008-2017: an ecological study \\ Integridad del sistema de información sobre tuberculosis en el estado de Paraná, 2008-2017: un estúdio \\ ecológico
}

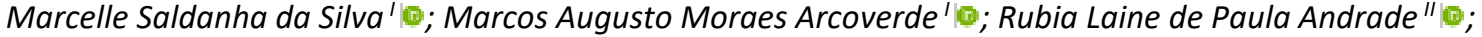

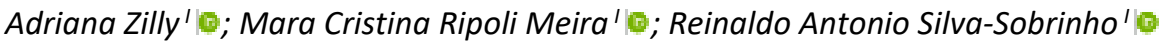

'Universidade Estadual do Oeste do Paraná, Foz do Iguaçu, PR, Brasil; "Universidade de São Paulo, Ribeirão Preto, SP, Brasil

\begin{abstract}
RESUMO
Objetivo: analisar a completude dos dados de tuberculose no Sistema de Informação de Agravos de Notificação (SINAN). Método: estudo ecológico composto por casos notificados de tuberculose tratados no Paraná de 2008 a 2017. Os dados foram agrupados em variáveis de notificação, acompanhamento e complementares e compreendiam informações de preenchimento obrigatórias e essenciais. Classificou-se a completude como excelente ( $<5 \%$ de incompletude), boa ( 5 a $<10 \%)$, regular (10 a $<20 \%$ ), ruim (20 a 50\%) e muito ruim (>50\%). A pesquisa atende a resolução 510/2016 do Conselho Nacional de Saúde. Resultados: variáveis obrigatórias apresentaram menos de $5 \%$ de incompletude, as essenciais tiveram avaliação muito ruim para teste de sensibilidade, tabagismo, drogas ilícitas. A completude do grupo acompanhamento piorou ao longo dos anos, demais grupos apresentaram melhora, exceto em 2014. Conclusão: completude insatisfatória em parte das variáveis essenciais e diminuição da completude no grupo acompanhamento revela repercussão no vinculo individuo-serviço de saúde e no desfecho dos casos.
\end{abstract}

Descritores: Sistemas de Informação em Saúde; Vigilância em Saúde Pública; Tuberculose; Notificação de Doenças.

\begin{abstract}
Objective: to examine the completeness of tuberculosis data in Brazil's Notifiable Disease Information System (SINAN). Method: this ecological study considered notified cases of tuberculosis treated in Paraná between 2008 and 2017. Data were grouped into notification, follow-up and complementary variables and comprised mandatory and essential information. Completeness was classified as excellent ( $<5 \%$ incomplete), good ( 5 to $<10 \%)$, regular $(10$ to $<20 \%)$, poor $(20$ to $50 \%$ ) and very poor $(>50 \%)$. The study complies with Conselho Nacional de Saúde Resolution 510/2016. Results: mandatory variables were less than 5\% incomplete, and essentials performed very poorly for sensitivity test, smoking and illicit drugs. The completeness in follow-up information worsened over the years, while other groups improved, except in 2014. Conclusion: unsatisfactory completeness in some essential variables, and decreasing completeness in follow-up information, reveal repercussions on bonding between individual and health service, and on case outcomes.

Descriptors: Health Information Systems; Public Health Surveillance; Tuberculosis; Disease Notification.

\section{RESUMEN}

Objetivo: examinar la integridad de los datos sobre tuberculosis en el Sistema de Información de Enfermedades Notificables de Brasil (SINAN). Método: este estudio ecológico consideró los casos notificados de tuberculosis tratados en Paraná entre 2008 y 2017. Los datos se agruparon en variables de notificación, seguimiento y complementarias y comprendieron información obligatoria y esencial. La integridad se clasificó como excelente ( $<5 \%$ incompleta), buena ( $5 \mathrm{a}<10 \%)$, regular ( $10 \mathrm{a}<20 \%)$, mala $(20$ a 50\%) y muy mala (> 50\%). El estudio cumple con la Resolución 510/2016 del Conselho Nacional de Saúde. Resultados: las variables obligatorias fueron menos del $5 \%$ incompletas, y lo esencial tuvo muy mal desempeño en la prueba de sensibilidad, tabaquismo y drogas ilícitas. La completitud en la información de seguimiento empeoró con los años, mientras que otros grupos mejoraron, excepto en 2014. Conclusión: completitud insatisfactoria en algunas variables esenciales, y una completitud decreciente en la información de seguimiento, revelan repercusiones en la vinculación entre individuo y servicio de salud, y en resultados del caso. Descriptores: Sistemas de Información en Salud; Vigilancia en Salud Pública; Tuberculosis; Notificatión de Enfermedades.
\end{abstract}

\section{INTRODUÇÃO}

Entre as doenças infectocontagiosas, a tuberculose se destaca como uma das que mais provoca mortes. Foram estimados 10 milhões de casos de tuberculose ao redor do mundo para o ano de 2018, com 1,2 milhões de óbitos em pessoas HIV negativas ${ }^{1}$. No Brasil, foram diagnosticados 72.788 novos casos da doença em 2018, com um coeficiente de incidência de 34,8 casos por 100 mil habitantes $^{2}$.

Em 2014, foi aprovado pela Organização Mundial de Saúde (OMS) a estratégia End TB (pelo fim da tuberculose) e, dentre os alvos da proposta, estão diminuir em $35 \%$ o número de mortes pela doença e reduzir em $20 \%$ a sua taxa de 
incidência até o ano 2020 comparado com 2015, e estipula como meta para o ano de 2030 o alcance da redução de $90 \%$ no número de mortes por tuberculose e $80 \%$ na taxa de incidência, a partir dos dados de $2015^{1,3}$. A redução cumulativa da taxa global de incidência no período de 2015-2018 foi de apenas 6,3\%, já a redução da mortalidade nesse mesmo período foi de $11 \%$, menos de um terço da meta proposta ${ }^{1}$, causando preocupação com a capacidade dos países em implementar a proposta e com a qualidade dos registros nos sistemas de informação em saúde.

Dentre as ações previstas para o controle da tuberculose, está o registro de informações dos casos, desde a busca inicial dos mesmos até o desfecho do tratamento. O registro oportuno e qualificado dos dados é um componente fundamental para o manejo dos casos e para o controle da doença, pois permite: monitorar as tendências da doença, o progresso do tratamento individual e coletivo, garantir a continuidade do cuidado quando é necessário o encaminhamento para outros estabelecimentos de saúde, monitorar o resultado do tratamento, além de permitir o planejamento e avaliação das estratégias do Programa Nacional de Controle da Tuberculose (PNCT) em seus diversos níveis ${ }^{4}$.

Dessa forma, os Sistemas de Informação em Saúde (SIS) são instrumentos significativos para a identificação da situação de saúde de uma determinada população, pois além de caracterizarem indivíduos em risco, auxiliam no direcionamento e planejamento de políticas públicas de acordo com as necessidades e singularidades de cada grupo populacional ${ }^{5}$. Para a utilização das informações registradas, é requisito manter uma alta qualidade dos dados, o qual deve ser regularmente mensurado, analisado e melhorado para atender as necessidades dos indivíduos ${ }^{6,7}$.

Um dos atributos integrantes da dimensão da qualidade dos dados diz respeito a completude das informações, que se refere à proporção de preenchimento das variáveis no sistema de notificação ${ }^{8-10}$.

Cabe ressaltar que a incompletude do registro de notificação e do acompanhamento dos pacientes com tuberculose pode trazer prejuízos à continuidade da assistência prestada, à troca de informações entre os diferentes serviços de atenção a saúde que assistem o paciente e, dificultar o acompanhamento e avaliação dos cuidados prestados $^{11}$, além de tornar questionável se a atividade assistencial que previa o registro não foi realizada de fato, ou se foi realizada e não foi registrada ${ }^{12}$, tornando difícil precisar se a falha está no preenchimento dos instrumentos ou no acompanhamento dos doentes ${ }^{13}$.

Diante do exposto, o presente estudo teve como objetivo analisar a completude dos dados referentes a tuberculose no Sistema de Informação de Agravos de Notificação (SINAN) do estado do Paraná, no período de 2008-2017.

\section{MÉTOdo}

Trata-se de um estudo epidemiológico, de abordagem ecológica com base em análise exploratória documental ${ }^{14}$, realizado no estado do Paraná, região sul do Brasil. O estado possui 399 municípios em uma área territorial de 199.305,236 km² e população estimada de 11.433 .957 pessoas com 74,9\% coberta pelas ações de atenção básica em saúde ${ }^{2,15}$, dispõe de um Índice de Desenvolvimento Humano (IDH) de 0,749, sendo considerado o 5 o melhor do país ${ }^{15}$.

A população do estudo foi composta pelos casos de tuberculose notificados no SINAN que realizaram o tratamento no estado do Paraná, no período de 2008 a 2017. Os dados foram fornecidos em julho de 2019, sendo disponibilizados pela Coordenação Geral do PNCT, mediante solicitação realizada pelo site do Sistema Eletrônico do Serviço de Informação ao Cidadão (e-SIC), disponível no endereço eletrônico: https://esic.cgu.gov.br/sistema/site/index.aspx.

A partir do banco de dados, foram selecionadas as variáveis necessárias para a realização de análises epidemiológicas e operacionais junto ao diagnóstico, acompanhamento e desfecho dos casos, fundamentais para que os serviços de saúde compreendam o comportamento da doença.

Todo caso diagnosticado de tuberculose deve ser obrigatoriamente notificado através da ficha de notificação/investigação do $\operatorname{SINAN}^{16}$, que compreende 47 variáveis divididas em quatro blocos, sendo eles: dados gerais, notificação individual, dados de residência e dados complementares. Os três primeiros blocos citados englobam informações referentes a unidade notificadora e ao indivíduo, como dados pessoais e de moradia. Já o último bloco contempla informações relacionadas à investigação do caso como tipo de entrada da pessoa no sistema de informação, forma da doença, pertencimento à população especial, resultados de exames, entre outros.

Para a atualização dos dados alusivos ao tratamento, utiliza-se o boletim de acompanhamento de caso que inclui informações referentes aos contatos examinados, realização das baciloscopias mensais, realização do Tratamento Diretamente Observado (TDO), situação de encerramento, etc.

De acordo com a ficha de notificação e boletim de acompanhamento, as variáveis foram selecionadas e divididas em três grupos, sendo eles: notificação (dados sociodemográficos), acompanhamento e complementar (proveniente do bloco dados complementares da ficha de notificação da tuberculose, compreendendo variáveis relacionadas ao histórico/condições clinicas do paciente e resultado de exames diagnósticos). 
As variáveis selecionadas também foram identificadas quanto ao tipo de preenchimento, em obrigatórias e essenciais (Figura 1). As variáveis obrigatórias são aquelas que, se não forem preenchidas não há como inserir a notificação no "SINAN", já as classificadas como essenciais são importantes para análises epidemiológicas e operacionais, porém, o seu não preenchimento não impede o registro do caso no Sistema de Informação ${ }^{16}$.

\begin{tabular}{|l|l|l|}
\hline \multirow{2}{*}{ Grupo das variáveis } & \multicolumn{2}{|c|}{ Variáveis e classificação de preenchimento } \\
\cline { 2 - 3 } & Obrigatórias & \multicolumn{1}{c|}{ Essenciais } \\
\hline Notificação & Sexo, gestante & Raça, escolaridade e zona de residência \\
\hline Acompanhamento & - & $\begin{array}{l}\text { Baciloscopia de segundo e sexto mês, tratamento diretamente observado (TDO) } \\
\text { indicado*, TDO realizado e situação de encerramento }\end{array}$ \\
\hline Complementar & $\begin{array}{l}\text { Tipo de entrada, forma } \\
\text { clínica, baciloscopia de } \\
\text { escarro diagnostica, } \\
\text { cultura de escarro, HIV }\end{array}$ & $\begin{array}{l}\text { Institucionalizado*, raio x de tórax, agravos associados [Aids, alcoolismo, } \\
\text { diabetes, doença mental, tabagismo, drogas ilícitas, outros], teste de } \\
\text { sensibilidade aos fármacos**, população especial [profissionais da saúde***, } \\
\text { população privada de liberdade***, população em situação de rua*** e } \\
\text { imigrantes***], benefícios do governo*** }\end{array}$ \\
\hline
\end{tabular}

*Variáveis preenchidas até 2014 no sistema de informação; **Variável preenchida a partir de 2014; ***Variáveis preenchidas a partir de 2015. FIGURA 1: Grupo de variáveis conforme o Sistema de Notificação de Agravos de Notificação e classificação quanto ao preenchimento obrigatória e essencial selecionadas para a pesquisa. Paraná, Brasil, 2019.

$\mathrm{Na}$ análise de dados, inicialmente utilizou-se o software $\mathrm{R}$ para o cálculo do percentual de incompletude de cada variável estudada para todo estado do Paraná, considerando as observações de todo período de estudo. Foi entendido como dado incompleto, os campos não preenchidos ("vazios") ou preenchidos como "ignorado". Estes últimos foram transformados para a informação "vazio" utilizando o software Microsoft Office Excel 2016. Dessa forma, a completude foi classificada da seguinte maneira: excelente (menos de 5\% de incompletude), bom (5-10\%), regular (10 a $20 \%)$, ruim (20 a 50\%) e muito ruim (acima de 50\%) $)^{17}$. Para evitar a sobreposição dos valores de incompletude nas categorias "bom", "regular" e "ruim", foram realizados ajustes nos pontos de corte, estipulando como bom (5 a <10\%) e regular (10 a $<20 \%$ ), como proposto por Lino e Fonseca ${ }^{18}$. Após essa classificação, calculou-se o percentual de incompletude de cada variável para cada ano de estudo e identificou-se o percentual mínimo e máximo de cada uma delas, indicando os anos em que estes ocorreram. A mediana do percentual de cada variável também foi calculada.

Por fim, calculou-se a média da incompletude de cada grupo de variáveis (notificação, acompanhamento e complementar), bem como do estado do Paraná por ano de estudo. Isso foi feito pela soma dos percentuais de incompletude das variáveis de cada grupo ou estado em cada ano estudado, dividindo-se pelo número de variáveis.

A pesquisa atende as determinações descritas na Resolução 466/2012 e 510/2016 do Conselho Nacional de Saúde e por tratar-se de dados de domínio público, com informação agregadas sem a possibilidade de identificação do indivíduo, torna-se dispensável a tramitação pelo Comitê de Ética em Pesquisa.

\section{RESULTADOS}

O estudo identificou 23.852 casos de tuberculose notificados que fizeram tratamento no estado do Paraná no período de 2008 a 2017. A completude de dados sobre tuberculose do SINAN no estado como um todo foi classificado como regular (10,1\% de incompletude). As variáveis categorizadas como obrigatórias obtiveram índices excelentes de completude (menor que $5 \%$ de incompletude). Relacionado às variáveis de preenchimento essencial, os menores valores de completude corresponderam aos campos Teste de Sensibilidade (TS) (61,0\% de incompletude), tabagismo (65,4 \% de incompletude) e drogas ilícitas (65,2 \% de incompletude) (Tabela 1). Nas variáveis de notificação, identificouse que o registro da escolaridade apresentou discreta melhora em 2016 quando comparado à 2010. Nas variáveis de acompanhamento, o registro da baciloscopia de controle (2 e 6ㅇ mês), bem como do TDO (tanto indicado quanto realizado) apresentou piora ao longo do tempo. E nas variáveis complementares, houve piora no registro da informação que diz respeito à condição de institucionalização do doente notificado e melhora no registo da Aids, tabagismo e drogas ilícitas (Tabela 1). 
TABELA 1: Completude das variáveis sobre tuberculose no Sistema de Informação de Agravos de Notificação (SINAN), de acordo com o percentual de incompletude. Paraná, 2008-2017.

\begin{tabular}{|c|c|c|c|c|c|}
\hline Variável & Incompletude (\%)* & Completude & Mín (ano)** & Mediana** & Max (ano)** \\
\hline \multicolumn{6}{|l|}{ Notificação } \\
\hline Sexo*** (obrigatória) & 0 & Excelente & 0 & 0 & 0 \\
\hline Gestante (obrigatória) & 0,8 & Excelente & $0,1(2017)$ & 0,7 & $1.9(2008)$ \\
\hline Raça & 2,5 & Excelente & $0,8(2016)$ & 2,7 & $3,4(2009 ; 2011)$ \\
\hline Escolaridade & 21,2 & Ruim & $17,4(2016)$ & 21,6 & $23,7(2010)$ \\
\hline Zona & 2,7 & Excelente & $1,4(2010)$ & 3,05 & 4,3 (2015) \\
\hline \multicolumn{6}{|l|}{ Acompanhamento } \\
\hline Baciloscopia 2o mês & 13,7 & Regular & $8,5(2010)$ & 13,8 & $20,1(2013)$ \\
\hline Baciloscopia 6ㅇ mês & 19 & Regular & $12,6(2008)$ & 20,45 & $26,5(2013)$ \\
\hline TDO indicado & 3,8 & Excelente & $0,8(2011)$ & 1,3 & $20,6(2014)$ \\
\hline TDO realizado & 2,4 & Excelente & $0,3(2008)$ & 0,65 & $9,9(2017)$ \\
\hline Situação de encerramento & 1,1 & Excelente & 0,0 (2010 a 2012) & 0,35 & $5,8(2017)$ \\
\hline \multicolumn{6}{|l|}{ Complementares } \\
\hline Tipo de entrada*** (obrigatória) & 0 & Excelente & 0 & 0 & 0 \\
\hline Institucionalizado & 4,4 & Excelente & $0,8(2012)$ & 1,9 & $21,0(2014)$ \\
\hline Raio x de tórax & 0,6 & Excelente & $0,2(2011)$ & 0,5 & $1,7(2017)$ \\
\hline Forma clínica (obrigatória) & 0,03 & Excelente & 0 (2008 a 2016) & 0 & $0,2(2017)$ \\
\hline Aids & 6,7 & Bom & $3,0(2014 ; 2015)$ & 4,3 & $16,1(2008)$ \\
\hline Alcoolismo & 3,4 & Excelente & $1,0(2012)$ & 2,75 & $7,9(2008)$ \\
\hline Diabetes & 3,6 & Excelente & $1,8(2012)$ & 2,7 & $9,00(2008)$ \\
\hline Doença mental & 3,5 & Excelente & $1,5(2012)$ & 2,95 & $8,40(2008)$ \\
\hline Tabagismo & 65,4 & Muito ruim & $2.2(2016)$ & 90,3 & $99,6(2009)$ \\
\hline Drogas ilícitas & 65,2 & Muito ruim & $2,8(2015)$ & 90,1 & $99,6(2009)$ \\
\hline Outros agravos & 8,4 & Bom & $5,5(2014)$ & 7,7 & $15,3(2008)$ \\
\hline $\begin{array}{l}\text { Baciloscopia de escarro para } \\
\text { diagnóstico (obrigatória) }\end{array}$ & 0,02 & Excelente & 0 (2008 a 2016) & 0 & $0,1(2017)$ \\
\hline Cultura (obrigatória) & 0,02 & Excelente & 0 (2008 a 2016) & 0 & $0,1(2017)$ \\
\hline Teste de sensibilidade & 61 & Muito ruim & $58,5(2016)$ & 62,25 & $72,2(2014)$ \\
\hline HIV (obrigatória) & 0,03 & Excelente & $\begin{array}{c}0(2008 \text { a 2013; 2015; } \\
\text { 2016) }\end{array}$ & 0 & $0,1(2014 ; 2017)$ \\
\hline Profissionais de saúde & 2,2 & Excelente & $1,1(2015 ; 2016)$ & 1,1 & $3,0(2017)$ \\
\hline PPL & 2,1 & Excelente & $1,1(2015 ; 2016)$ & 1,1 & $2,6(2017)$ \\
\hline População de rua & 2,3 & Excelente & $1,0(2015)$ & 1,2 & $3,1(2017)$ \\
\hline Imigrantes & 2,3 & Excelente & $1,1(2015)$ & 1,2 & $3,1(2017)$ \\
\hline Beneficiários do governo & 1,8 & Excelente & $1,4(2015)$ & 1,5 & $3,5(2017)$ \\
\hline
\end{tabular}

Legenda: TDO - Tratamento Diretamente Observado; PPL - pessoa privada de liberdade.

* percentual de incompletude de cada variável, calculado considerando todas as observações do estado do Paraná para todo período de estudo;

**valor mínimo, mediana e valor máximo foi identificado considerando o cálculo anual da incompletude de cada variável.

A análise da completude ao longo dos anos apontou que o conjunto das variáveis de notificação sustentou uma completude boa/excelente durante o período, já o grupo acompanhamento apresentou piora na completude ao longo dos anos, saindo da classificação excelente (4,7\%) no ano de 2008 para regular (14,5\%) em 2017.

O preenchimento do grupo das variáveis complementares atingiu a classificação ruim (21,7\%) no ano de 2014 , evoluindo com classificações mais satisfatórias ao longo do tempo (Figura 2). 


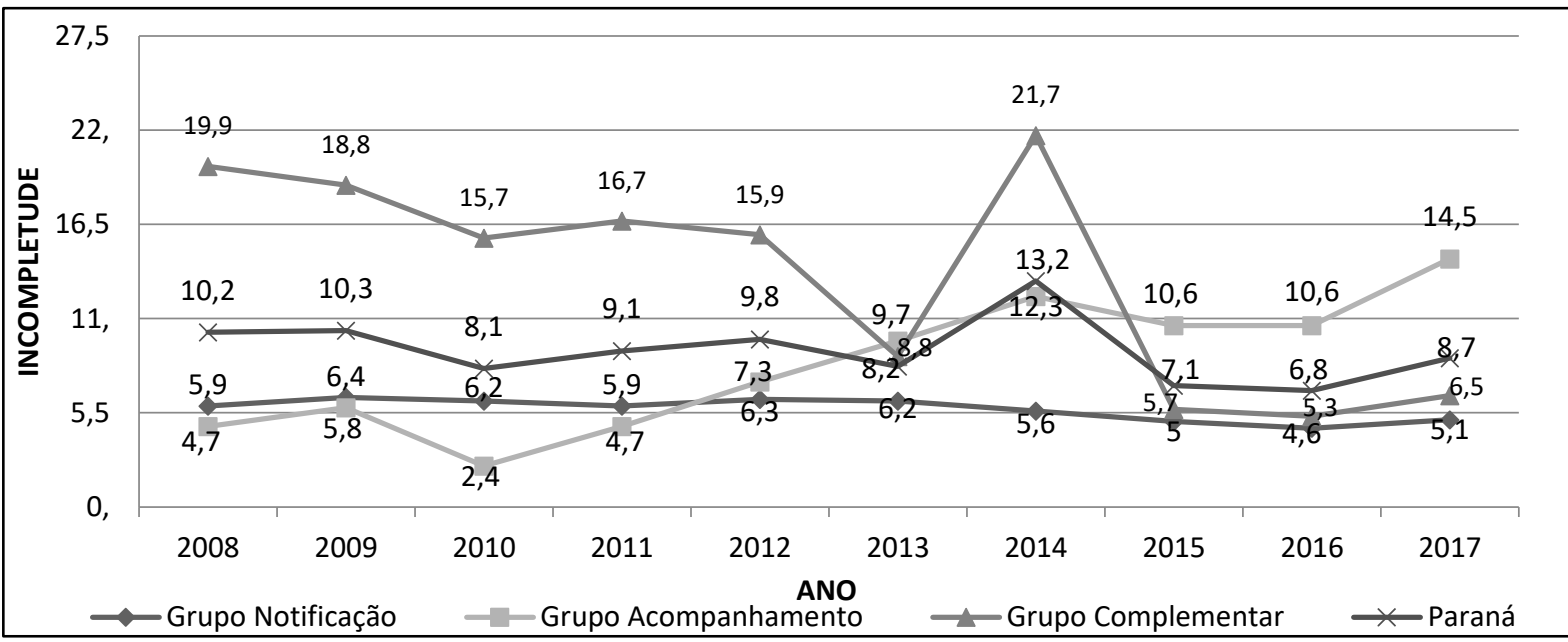

FIGURA 2: Percentual de incompletude das variáveis de tuberculose do Sistema de Informação de Agravos de Notificação do estado do Paraná, segundo os grupos de variáveis. Paraná, Brasil, 2008-2017.

\section{DISCUSSÃO}

A qualidade dos dados em saúde é um atributo importante por proporcionar o entendimento do comportamento das doenças em um determinado espaço geográfico, além de auxiliar no planejamento, implementação e avaliação das ações em saúde ${ }^{19,10}$.

Durante o período avaliado, o estado do Paraná apresentou completude geral regular para tuberculose conforme o banco de dados do SINAN. Destaca-se que, a falta de preenchimento adequado das variáveis pode estar relacionada com a falta de envolvimento dos profissionais responsáveis pela notificação, desconhecimento sobre a importância das informações a ser coletada, concebendo tal atividade como mera atribuição burocrática, sem vínculo com a qualidade do serviço ${ }^{20}$; desconhecimento do fluxo das informações no sistema ${ }^{19}$ ou ainda sobrecarga dos profissionais de saúde que canalizam seu tempo para atividades tidas como prioritárias segundo seus julgamentos 5 .

Os dados do SINAN que foram gerados nos municípios devem ser transferidos semanalmente pelas secretarias municipais de saúde para as secretarias estaduais, e estas devem enviar quinzenalmente os dados estaduais para a Secretaria de Vigilância em Saúde (SVS/MS), ficando a nível de cada unidade federada a responsabilidade pela qualidade dos dados transmitidos ${ }^{16}$. Nas buscas realizadas na literatura, não foram encontradas a nível estadual ou dos municípios do estado do Paraná, programa que contemple o controle de qualidade dos dados dos SIS.

Compete a União, aos estados e municípios, prezar pela qualidade das bases de dados de saúde, cada qual possui um conjunto de atribuições e responsabilidades ${ }^{16}$. Para o processo contínuo de controle da qualidade dos dados, parece coerente a averiguação da completude dos dados em intervalos regulares, a expedição de relatórios técnicos de acompanhamento, capacitação dos profissionais de saúde, visitas técnicas continuadas de caráter educativo para fortalecimento do vínculo e a co-responsabilização entre níveis administrativos visando à gestão, à estruturação e à operacionalização do SINAN.

A completude classificada como excelente para as variáveis obrigatórias já era esperada pois a inexistência desses dados impossibilita a inserção da notificação no SINAN. Houve variação de excelente a muito ruim nas variáveis essenciais, que são aquelas que não possuem obrigatoriedade no seu preenchimento, porém, são importantes para cálculos de indicadores epidemiológicos e operacionais ${ }^{16}$, como é o caso do TS, tabagismo e drogas ilícitas.

A oferta universal do TS é um componente do pilar de prevenção e cuidado integrado e centrado no paciente do Plano Nacional pelo Fim da TB que, embasado no modelo proposto pela OMS, visa reduzir a incidência da doença para menos de 10 casos por 100 mil habitantes e o coeficiente de mortalidade para menos de 1 óbito por 100 mil habitantes até o ano de $2035^{21}$. O prejuízo da oferta do TS acarreta atraso no diagnóstico dos casos que cursam com resistência e o emprego de esquema de tratamento ineficaz ${ }^{22}$. Estudo anterior ${ }^{22}$ apontou que a mediana de tempo entre a confirmação do diagnóstico de tuberculose e a detecção da resistência foi de 12 meses, o que contribui para a manutenção da cadeia de transmissão do bacilo resistente e agravo das condições clínicas do paciente.

Indicadores de vulnerabilidade social, dentre eles o uso de drogas lícitas ou ilícitas, dificultam a criação de vínculo satisfatório com o serviço de saúde e são preditores de maiores riscos de abandono do tratamento 23,24 , o que torna preocupante a baixa completude de dados para essas variáveis. Indivíduos tabagistas possuem chances $70 \%$ maiores de 
desfecho negativo no tratamento da tuberculose quando comparados com aqueles que não fumam ${ }^{25}$. Além disso, o tabagismo se associa com atraso no diagnóstico da doença ${ }^{26}$, falha do tratamento ${ }^{27}$, recidiva e maiores chances de óbito ${ }^{28,29}$, reforçando a importância do registro correto dos dados nos SIS.

Quanto a classificação das variáveis por grupo, o de notificação foi o único que manteve completude boa/excelente durante o período avaliado, possivelmente por abarcar um maior percentual de variáveis obrigatórias. A completude satisfatória nesse grupo é importante pois variáveis como raça, sexo e escolaridade contribuem para a identificação de indivíduos que apresentem maiores chances de desfechos negativos, haja visto que são fatores preditivos associados a falência do tratamento, abandono e óbito ${ }^{13,30}$. Nesse grupo de variáveis, a melhora no preenchimento de informações sobre a escolaridade dos individuos ao longo do tempo pode permitir a prestação de um cuidado adequado aos mesmos, uma vez que a depender da escolaridade, o entendimento em relação ao processo saúde-doença são diferentes, bem como a capacidade de compreender e seguir as orientações sobre a doença e o tratamento ${ }^{31}$.

Apesar do grupo acompanhamento dispor de uma média de completitude classificada como boa para o período estudado, esse grupo passou de uma completude excelente nos anos iniciais do estudo, transitando por completude classificada como boa e para regular nos anos finais, e caso esse padrão se mantenha, futuramente poderá haver impacto negativo no desfechos positivos dos casos da doença.

A classificação regular da completude nas variáveis do grupo acompanhamento a partir do ano de 2014, pode indicar um descuido no preenchimento do sistema de informação em função dessas variáveis não serem de preenchimento obrigatório, bem como um seguimento insatisfatório dos casos principalmente no que diz respeito à realização das baciloscopias de controle, cujo preenchimento apresentou piora ao longo do tempo. Ainda no grupo acompanhamento, também pode-se supor uma fragilidade no vínculo entre o indivíduo e o serviço de saúde, o qual tem grande potencial de ser proporcionado pelo $\mathrm{TDO}^{32}$. O vínculo criado entre o paciente e o serviço de saúde é de grande importância para a continuidade do tratamento da tuberculose, pois vínculos estreitos e boa comunicação permite que o indivíduo se sinta mais seguro para explanar suas dúvidas e anseios ${ }^{31}$. A comunicação bem estabelecida entre o indivíduo e os profissionais de saúde é essencial para evitar o abandono do tratamento.

Estudo anterior ${ }^{32}$ evidenciou que indivíduos com acompanhamento insuficiente realizado pelos serviços de saúde estavam associados mais fortemente ao abandono, complicações e óbito. A perda de seguimento dos pacientes pode elevar as taxas de notificação e de casos de tuberculose recorrente e de infecção prolongada, aumentar os casos de resistência, assim como elevar as taxas de morbidade e mortalidade da doença ${ }^{33}$.

Pacientes com histórico de abandono do tratamento possuem determinantes sociais que aumentam as chances dessa escolha vir a se repetir, sendo necessário que os profissionais de saúde realizem um acompanhamento robusto dos pacientes com a doença, adequando o tratamento a realidade dos mesmos, a fim de minimizar o risco de desfechos negativos ${ }^{34}$.

A alteração na ficha de notificação da tuberculose no ano de 2014 pode ter impactado na completude das variáveis do grupo complementar no referido ano, uma vez que o registro destas vinham indicando melhora ao longo do tempo mesmo com esse grupo apresentando três quartos de variáveis de preenchimento essencial. A modificação resultou na retirada das variáveis ocupação, indivíduo institucionalizado, teste tuberculínico, baciloscopia e cultura de outro material além do escarro, drogas utilizadas no tratamento, indicação para o TDO e se a doença estava relacionada ao trabalho e na inclusão das seguintes variáveis: pós-óbito no tipo de entrada do indivíduo no sistema, populações especiais, beneficiários do governo, uso de drogas ilícitas e tabagismo no campo doenças e agravos associados, uso de terapia antirretroviral durante o tratamento, resultado do teste rapido molecular para tuberculose (TRM-TB) e resultado do TS.

A modificação na ficha de notificação da doença abarcou parte das variáveis constituintes do grupo complementar que apresentou piora na completude dos dados no ano de 2014, provavelmente relacionado com a implantação e adaptação com a nova ficha nos serviços de saúde e com o período de transição da ficha antiga para a atual. Além disso, nesse ano foi possível observar alta proporção de incompletude na variável institucionalizado (último ano da variável no sistema) e teste de sensibilidade (variável nova), bem como nos dados sobre uso de drogas $-42,3 \%$ e tabaco - 43,6\% (dados não mostrados). Acredita-se que o registro do teste de sensibilidade não é feito em função de nem todos os pacientes realizarem o mesmo, bem como pelo fato do resultado ser demorado, levando os profissionais a esquecerem de registrar tal informação ${ }^{35}$. Quanto ao preenchimento dos dados sobre o uso de substancias psicoativas, estas também podem ajudar no delineamento do tratamento dos doentes de TB, de tal modo que os profissionais de saúde que atuam na atenção à doença precisam fazer uma interlocução com os serviços psiquiátricos, de redução de danos e até mesmo com o serviço social para uma atenção integral a esses indivíduos, visto as dificuldades enfrentadas para promover a adesão ao tratamento deste grupo de pacientes ${ }^{23}$.

Ainda no grupo de variáveis complementares, é possível observar uma melhora no registro da informação Aids ao longo do tempo, levantando-se a hipótese de que tal melhora acompanha o processo de desmistificação da doença ao 
longo do tempo ou uma maior sensibilização dos profissionais de saúde quanto à importância de realizar esse tipo de registro para a definição adequada do tratamento medicamentoso.

\section{Limitações do estudo}

É importante considerar as seguintes limitações do estudo: tipo de estudo, o qual não permite traçar considerações sobre municípios específicos do estado; um possível viés de informação por utilizar de dados secundários; opção por uma visão geral da completude do Sistema de Informação em Tuberculose do estado no Paraná, agrupando variáveis de preenchimento obrigatório e essenciais, o que metodologicamente pode ser questionado (embora a presença dessas variáveis nos grupos tenha sido devidamente ponderada na presente discussão).

\section{CONCLUSÃO}

A completude insatisfatória identificada no banco de dados do SINAN referente a tuberculose no estado do Paraná pode acarretar dados não fidedignos ao perfil epidemiológico da doença e causar dificuldades na sua utilização como ferramenta para a formulação de políticas públicas de saúde e para a avaliação de ações implementadas para a prevenção e controle da doença.

Ressalta-se a importância dos estados e municípios dispor de um programa de controle de qualidade dos dados nos SIS e a contínua capacitação dos servidores da saúde relacionado a relevância do preenchimento adequado dos campos do sistema, que se traduz não como mera atribuição burocrática destituída da assistência, mas sim como um potencializador de uma ferramenta de grande valor para o planejamento de políticas públicas em saúde.

\section{REFERÊNCIAS}

1. World Health Organization. Global Tuberculosis Report 2019. Geneva: WHO; 2019. [cited 2020 Jan 02]. Available from: https://www.who.int/teams/global-tuberculosis-programme/tb-reports/global-report-2019.

2. Ministério da Saúde (Br). Secretaria de Vigilância em Saúde. Brasil livre da tuberculose: evolução dos cenários epidemiológicos e operacionais da doença. Brasília (DF): Ministério da Saúde; 2019. Available from: https://portalarquivos2.saude.gov.br/images/pdf/2019/marco/22/2019-009.pdf.

3. Barreira D. The challenges to eliminating tuberculosis in Brazil. Epidemiol. Serv. Saude [Internet], 2018 [cited 2020 Jan 02]; 27(1):1-4. DOI: https://doi.org/10.5123/s1679-49742018000100009.

4. World Health Organization. Electronic recording and reporting for tuberculosis care and control. Geneva: WHO, 2012. [cited 2020 Jan 02]. Available from: https://www.who.int/tb/publications/electronic_recording_reporting/en/.

5. Zilmer JGV,Schwartz E, Muniz RM, Lima LM. Evaluation of the completeness of informations of hyperday in a Basic Unit of South of Brazil. Rev. Gaucha Enferm. [Internet], 2010 [cited 2020 Jan 02]; 31(2):240-6. DOI: https://doi.org/10.1590/S198314472010000200006.

6. Calazans ATS. Informations quality: concepts and applications. Transinformação [Internet], 2008 [cited 2020 Jan 06]; 20(1):2945. DOI: https://doi.org/10.1590/S0103-37862008000100003

7. Cai L, Zhu Y. The challenges of data quality and data quality assessment in the big data era. Data Science Journal [Internet], 2015 [cited 2020 Jan 06]; 14(2):1-10. DOI: http://doi.org/10.5334/dsj-2015-002.

8. Doyle TJ, Glynn MK, Groseclose SL. Completeness of notifiable infections disease reporting in the United States: an analytical literature review. Am. J. Epidemiol. [Internet]; 2002 [cited 2020 Jan 06]; 155(9):866-874. DOI: https://doi.org/10.1093/aje/155.9.866.

9. World Health Organization. Comunicable disease surveillance and response systems: guide to monitoring and evaluating. Geneva: WHO, 2006. [cited 2020 Jan 06]. Available from: https://www.who.int/csr/resources/publications/surveillance/WHO_CDS_EPR_LYO_2006_2/en/.

10. Braz RM, Tauil PL, Santelli ACFS, Fontes CJF. Evaluation of the completeness and timeliness of malaria reporting in the Brazilian Amazon, 2003-2012. Epidemiol. Serv. Saúde [Internet]; 2016 [cited 2020 Jan 12]; 25 (1):21-32. DOI: http://dx.doi.org/10.5123/s1679-49742016000100003.

11. Orfao NH, Crepaldi NY, Brunello MEF, Andrade RLP, Monroe AA, Ruffino-Neto A et al. Coordinated care for tuberculosis: data registration and implementation of a computerized system. Ciencia \& Saúde Coletiva [Internet]; 2017 [cited 2020 jan 12]; 22(6):1969-77. DOI: https://doi.org/10.1590/1413-81232017226.15352016.

12. Coelho TTG, Medeiros ACQ, Ribeiro WCS, Menezes TB. Evaluation of the Level of Completeness of Maternity Cards from Postpartum Mothers Assisted in a University Hospital. R. Bras. Ci. Saúde [Internet]; 2015 [cited 2020 Jan 12]; 19 (2):117-22. DOI: https://doi.org/10.4034/RBCS.2015.19.02.05.

13. Malhão TA, Oliveira GP, Codennoti SB, Moherdaul F. Evaluation of data completeness in the Tuberculosis Notification Information System, Brazil, 2001-2006. Epidemiol. Serv. Saude [Internet]. 2010 [cited 2020 Jan 12]; 19(3): 245-56. Available from: http://scielo.iec.gov.br/scielo.php?script=sci_abstract\&pid=S1679-49742010000300007\&lng=en\&nrm=iso\&tlng=en.

14. Morgenstern H. Ecologic Studies in Epidemiology: concepts, principles and methods. Annu Rev Public Health [Internet]; 1995 [cited 2020 jan 30]; 16:61-81. DOI: https://doi.org/10.1146/annurev.pu.16.050195.000425.

15. Instituto Brasileiro de Geografia e Estatística (Br). Paraná: panorama geral. [cited 2020 Jan 12]. Available from: https://cidades.ibge.gov.br/brasil/pr/pesquisa/23/27652?detalhes=true. 
16. Ministério da Saúde (Br). Secretaria de Vigilancia em Saúde. Departamento de Vigilancia Epidemiológica. Sistemas de Informação de Agravos de Notificação. Normas e rotinas. Brasília (DF): Ministério da Saúde, 2006.

17. Romero DE, Cunha CB. Evaluation of quality of epidemiological and demographic variables in the Live Births Information System, 2002. Cad. Saúde Pública [Internet]; 2007 [cited 2020 Jan 12]; 23 (3): 701-14. DOI: http://dx.doi.org/10.1590/S0102$311 \times 2007000300028$.

18. Lino RRG, Fonseca SC, Kale PL, Pinheiro RS, Coeli CM. Trend of incompleteness of vital statistics in the neonatal period, State of Rio de Janeiro, Brazil, 1999-2014. Epidemiol. Serv. Saúde [Internet]; 2019 [cited 2020 Jan 14]; 29(2):e2018131. DOI: https://doi.org/10.5123/s1679-49742019000200014.

19. Alkhalawi MJ, Mcnabb SJN, Assiri AM, Memish ZA. Evaluation of tuberculosis public health surveillance, Al-Madinah province, Kingdom of Saudi Arabia, 2012. Journal of Epidemiology and Global Health [Internet]; 2016 [cited 2020 Jan 14]; 6(1):37-44. DOI: https://doi.org/10.1016/j.jegh.2015.04.005.

20. Santos NP, Lírio M, Passos LAR, Dias JP, Kritski Al, Galvão-Casto B, et al. Completeness of tuberculosis reporting forms in five Brazilian capitals with a high incidence of the disease. J. Bras. Pneumol. [Internet]; 2013 [cited 2020 Jan 18]; 39 (2): 221-5. DOI: https://doi.org/10.1590/S1806-37132013000200014.

21. Ministério da Saúde (Br). Secretaria de Vigilancia em Saúde. Boletim epidemiológico. Implantação do plano nacional pelo fim da tuberculose como problema de saúde pública no Brasil: primeiros passos rumo ao alcance das metas. Brasila (DF): Ministério da Saúde, 2018.

22. Savioli MTG, Morrone N, Santoro I. Primary bacillary resistance in multidrug-resistant tuberculosis and predictive factors associated with cure at a referral center in São Paulo, Brazil. J. Bras Pneumol. [Internet]; 2019 [cited 2020 Jan 18]; 4(2):e20180075. DOI: https://doi.org/10.1590/1806-3713/e20180075.

23. Sá AMM, Santiago LA, Santos NV, Monteiro NP, Pinto PHA, Lima AM et al. Reasons for treatment abandonment among tuberculosis patients. Rev. Soc. Bras. Clin. Med. [Internet]; 2017 [cited 2020 Jan 18]; 15 (3): 155-60. Available from: http://docs.bvsalud.org/biblioref/2017/11/875434/sbcm_153_155-160.pdf.

24. Ferreira MRL, Bonfim RO, Siqueira TC, Orfão NH. Abandonment of tuberculosis treatment: an integrative review. Rev. Enferm. Contemp. [Internet]; 2018 [cited 2020 Jan 18]; 7(1):63-71. DOI: http://dx.doi.org/10.17267/2317-3378rec.v7i1.1579.

25. Gegia M, Magee MJ, Kempker RR, Kalandadze I, Chakhaia T, Golub JE, et al. Tobacco smoking and tuberculosis treatment outcomes: a prospective cohort study in Georgia. Bull World Health Organ. [Internet]; 2015 [cited 2020 Jan 20], 93:390-9. DOI: http://dx.doi.org/10.2471/BLT.14.147439.

26. Rabin AS, Kuchukhidze G, Sanikidze E, Kempker RR, Blumberg HM. Prescribed and self-medication use increase delays in diagnosis of tuberculosis in the country of Georgia. Int. J. Tuberc. Lung. Dis. [Internet]; 2013 [cited 2020 Jan 20]; 17(2):214-20. DOI: http://dx.doi.org/10.5588/ijtld.12.0395.

27. Tachfouti N, Nejjari C, Benjelloun MC, Berraho M, Elfakir S, El Rhazi K, et al. Association between smoking status, other factors and tuberculosis treatment failure in Morocco. Int. J. Tuberc. Lung. Dis. [Internet]; 2011 [cited 2020 Jan 20]; 15(6):838-43. DOI: https://doi.org/10.5588/ijtld.10.0437.

28. Leung CC, Yew WW, Chan CK, Tam CM, Lam CW, Chang KC, et al. Smoking and tuberculosis in Hong Kong. Int. J. Tuberc. Lung. Dis. [Internet]; 2003 [cited 2020 Jan 20]; 7(10):980-6. Available from: https://pubmed.ncbi.nlm.nih.gov/14552569/.

29. Gupta PC, Pednekar MS, Parkin DM, Sankaranarayanan R. Tobacco associated mortality in Mumbai (Bombay) India. Results of the Bombay cohort study. Int. J. Epidemiol. [Internet]; 2005 [cited 2020 Jan 22]; 34(6):1395-1402. DOI: https://doi.org/10.1093/ije/dyi196.

30. Durans JJF, Sá EM, Pereira LFB, Soares DL, Oliveira PS, Aquino DMC, et al. Clinical and sociodemographic profile of patients who abandoned tuberculosis treatment in São Luís, Maranhão, Brasil. Rev. Pesq. Saúde [Internet]; 2013 [cited 2020 Jan 22]; 14(3):175-8. Available from: http://www.periodicoseletronicos.ufma.br/index.php/revistahuufma/article/view/2794/4075.

31. Brunello MEF, Cerqueira DF, Pinto IC, Arcenio RA, Gonzales RIC, Villa TCS, et al. Interaction between patient and health care professionals in the management of tuberculosis. Acta Paul. Enferm. [Internet], 2009 [cited 2020 Jan 22]; 22(2):176-82. DOI: https://doi.org/10.1590/S0103-21002009000200010.

32. Bastas PC, Marques M, Oliveira RL, Cunha EAT, Resendes APC, Souza-Santos, R. Social inequalities and tuberculosis: an analysis by race/color in Mato Grosso do Sul, Brazil. Rev. Saude Pública. [Internet]; 2013 [cited 2020 Jan 22]; 47(5): 854-64. DOI: https://doi.org/10.1590/S0034-8910.2013047004628.

33. Liaw YC, Richard A, Mohammad SJ, Valentine JG. Factors contributing towards loss to follow up among tuberculosis patients in Sabah. Borneo Journal of Medical Sciences (BJMS) [Internet]; 2019 [cited 2020 Feb 08]; 2:9-10. Avaliable from: https://jurcon.ums.edu.my/ojums/index.php/bjms/article/view/1796.

34. Tatés-Ortega N, Álvarez J, López L, Mendoza-Ticona A, Alarcón-Arrascue E. Loss to follow-up in patients treated for multidrugresistant tuberculosis in Ecuador. Rev. Panam. Salud Publica [Internet]; 2019 [cited 2020 Jan 24]; 43(91). DOI: https://doi.org/10.26633/RPSP.2019.91.

35. Lopes LN, Cardoso LL, Silva MS, Tonin E, Zilly A, Silva-Sobrinho RA. Rapid molecular assay for tuberculosis: cost and contributions. Rev. baiana enferm. [Internet]; 2020 [cited 2020 May 06]; 34:e34803.

DOI: http://dx.doi.org/10.18471/rbe.v34.34803. 